\title{
ON THE POSSIBILITY OF REALIZING SHORTEST BUNCHES IN LOW-ENERGY STORAGE RINGS
}

\author{
A.I.Papash $^{\text {a,e\# }}$, A.A. Alzeanidi ${ }^{\mathrm{d}}$, M. El Ghazaly ${ }^{\mathrm{d}}$, K.-U. Kühnel ${ }^{\mathrm{a}}$, C.P.Welsch ${ }^{\mathrm{a}, \mathrm{b}, \mathrm{c}}$ \\ ${ }^{a}$ Max Planck Institute for Nuclear Physics, 69117 Heidelberg, Germany \\ ${ }^{b}$ Kirchhoff Institute for Physics, University of Heidelberg, 69120 Heidelberg, Germany \\ ${ }^{c}$ Gesellschaft für Schwerionenforschung, 64291 Darmstadt, Germany \\ ${ }^{\mathrm{d}}$ KACST, 11442 Riyadh, Saudi Arabia \\ ${ }^{\mathrm{e} J o i n t ~ I n s t i t u t e ~ f o r ~ N u c l e a r ~ R e s e a r c h, ~ D u b n a, ~ R u s s i a, ~ o n ~ l e a v e ~}$
}

\begin{abstract}
For some very interesting experiments in future lowenergy storage rings it is highly desirable to realize ultrashort bunches in the nanosecond regime. These bunches could then be used for collision studies with atomic or molecular gas jet targets where the time structure of the bunches would be used as a trigger for the experiment. Thus, the control of the longitudinal time structure of the stored beam is of central importance since it directly determines the quality of the envisaged experiments. Since many years it has been a significant challenge for the storage ring accelerator-physics community to develop techniques to reduce the duration of bunches. Up to now, all methods that have been developed go along with various difficulties, which can include reduced stored-beam lifetimes. Thus, novel and innovative concepts for the manipulation and control of the longitudinal beam structure will have to be developed. In this paper, a possible approach to realize shortest bunches in an electrostatic storage ring is presented.
\end{abstract}

\section{INTRODUCTION}

Low-energy antiprotons are the ideal and perhaps the only tool to study in detail correlated quantum dynamics of few-electron systems in the sub-femtosecond time regime. Unfortunately cooled beams of antiprotons with the necessary beam quality and luminosity are not yet available and cannot be provided with present scientific infrastructures. In order to pave the way for a nextgeneration low-energy antiproton facility, challenging developments in both, storing and imaging techniques have been launched at the Max Planck Institute for Nuclear Physics (MPI-K). A novel ultra-low energy storage ring (USR) $[1,2]$ to be integrated at the proposed facility for low-energy antiproton and ion research (FLAIR) [3,4] is being developed to provide electroncooled beams of antiprotons and possibly highly charged ions in the energy range between 300 and $20 \mathrm{keV} / \mathrm{q}$, maybe even approaching the sub $\mathrm{keV}$ regime.

To allow for kinematically complete investigations of a variety of different collision processes, a reaction microscope shall be integrated in the ring thus achieving unprecedented luminosities [1,5]. For these collision experiments around $10^{4}$ antiprotons are required per bunch. This number is well below the space charge limit

\footnotetext{
\#Alexander.Papash@mpi-hd.mpg.de
}

04 Hadron Accelerators of the USR even at the lowest energies. However, the time structure of the pulses needs to be in the order of a few nanoseconds to allow its use as a trigger signal for the measurements. In this respect, an efficient control of the longitudinal time structure of the stored ion beam is of central importance since it directly determines the quality of the envisaged experiments.

\section{GENERAL APPROACH TO SHORT PULSES OPERATION}

It is technically possible to provide ultra-short pulses of $20 \mathrm{keV}$ antiprotons by manipulation of the time structure of an extracted beam. The beam will interact with e.g. a gas jet only once per cycle which takes $\sim 5 \mathrm{~s}$ in the case of the USR. This way a sufficiently high luminosity as required by the experiments cannot be reached.

We therefore consider the possibility of producing ultra-short pulses of a beam circulating in the USR [6]. It is clearly impossible to create ultra-short bunches of a few ns duration in one step from a coasting beam. With a revolution period of $20 \mu$ s the required buncher voltage to provide a $2 \mathrm{~ns}$ time focus would exceed $10 \mathrm{kV}$ and thus the induced energy spread by the phase compression would simply destroy the beam circulating in the ring.

An operation with ultra-short pulses for in-ring experiments might be realized by applying the sequence of the following procedures: Once the beam has been slowed down to $20 \mathrm{keV}$, the coasting beam of antiprotons is cooled down to a momentum spread of $\sim 10^{-4}$ [7]. Then the cooled beam is adiabatically captured into $\tau \sim 50 \mathrm{~ns}$ stationary buckets formed by a $20 \mathrm{MHz}$ cavity operating at a high harmonic mode of the ring revolution frequency $\left(h_{R F}=300-400\right)$, see table 1 .

The desired ultra-short pulses of $\sim 2$ ns duration will then be formed in the symmetry point of the straight section where the reaction microscope is located. The focus will be provided by an additional $3 \beta \lambda / 2$ double drift buncher, placed at the beginning of the straight section. Once the experimental section is crossed, a debuncher will provide phase decompression and limit the growth of the equilibrium momentum spread. Otherwise the increasing energy spread introduced by the phase compressor would cause a beam blow up in the bending sections of the ring. 
Table 1: High harmonic mode of the USR

\begin{tabular}{|c|c|c|}
\hline \multirow[t]{2}{*}{ Parameter } & \multicolumn{2}{|c|}{$\begin{array}{c}8^{\circ}+37^{\circ}+37^{\circ}+8^{\circ} \\
\text { deflectors }\end{array}$} \\
\hline & Injection & Extraction \\
\hline Energy $[\mathrm{keV}]$ & 300 & 20 \\
\hline Circumference $[\mathrm{m}]$ & 42.984 & 42.984 \\
\hline$\beta=v / c$ & $2.53 \cdot 10^{-2}$ & $6.53 \cdot 10^{-3}$ \\
\hline Rotation period $T=L / \beta c[\mu \mathrm{s}]$ & 5.667 & 21.957 \\
\hline Rotation frequenc, $F_{r o t}[\mathrm{kHz}]$ & 176.46 & 45.5436 \\
\hline RF frequency $F_{R F}[\mathrm{MHz}]$ & 20.1164 & 20.0392 \\
\hline RF harmonic number $h_{R F}$ & 114 & 440 \\
\hline Bucket RF width [ns] & 49.71 & 49.9 \\
\hline Buncher drift space $[\mathrm{cm}]$ & 200 & 200 \\
\hline Buncher voltage $[\mathrm{kV}]$ & 21.5 & 0.37 \\
\hline Expected Pulse width [ns] & 2 & 2 \\
\hline Momentum spread (before / & $5 \cdot 10^{-3}$ & \\
\hline after e-cooling) & $5 \cdot 10^{-4}$ & $5 \cdot 10^{-4}$ \\
\hline Achromatic mode & 0.7 & 0.7 \\
\hline dispersion $D_{\max } / D_{\min }[\mathrm{m}]$ & 0 & 0 \\
\hline$d P / P[\%]$ & 0.5 & $\leq 2$ \\
\hline Transition factor $\gamma_{t r}$ & & 2.2 \\
\hline Momentum compaction $\alpha$ & & 0.207 \\
\hline Frequency slip factor $\eta$ & & -0.8 \\
\hline Synchr. freq. $\Omega_{S}[\mathrm{kHz}]$ & & $1.7 \times \mathrm{V}_{\mathrm{RF}}^{1 / 2}$ \\
\hline Range of RF voltage change & & $10 \ldots 2000$ \\
\hline Momentum $p c[\mathrm{MeV}]$ & 23.72 & 6.12 \\
\hline $\begin{array}{l}\text { Time-of-flight } \tau_{e f} \text { in the } \\
\text { buncher gap } 20 \mathrm{~mm} \text { [ns] }\end{array}$ & $2.6\left(19^{\circ}\right)$ & $10\left(70^{\circ}\right)$ \\
\hline$\beta$ - (at buncher location) [m] & & 5 \\
\hline
\end{tabular}

As it was already pointed out, the phase compression will lead to an additional energy spread. This requires that any manipulation of the beam towards short pulses needs to be limited to the straight sections of the ring, where the dispersion function needs to be zero. To allow for this special operation mode, the USR lattice was recently modified substantially [6].

While the original layout of the USR foresaw $90^{\circ}$ bending elements in each of the ring's corner section [3,8], a modified design was proposed in 2006 [1]. This layout, however, is under reconsideration and the ring parameters for the short pulse operation mode are presented in the above table 1 . The RF phase gymnastics will be applied to the antiproton beam at lowest energy.

\section{LOW-ENERGY PHASE COMPRESSOR}

The estimation of the parameters of the USR phase compressor as well as the expected pulse structure of the beam as presented in this paper are calculated with the computer code Buncher U400 [9]. We applied a sinusoidal voltage at $20 \overline{\mathrm{MHz}}$ to the phase compressor and assumed that the beam has already been pre-bunched to $25 \mathrm{~ns}$ pulses by adiabatic capture into RF buckets. We furthermore assumed that the high harmonic RF will keep

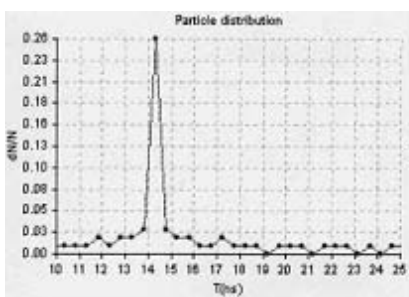

a)

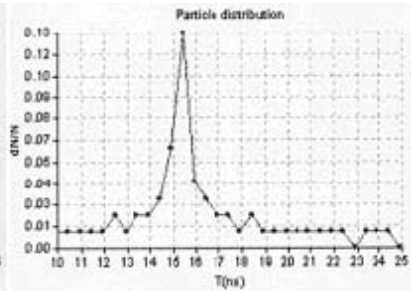

b)
Figure 1: a) Beam pulses at an RF frequency of $f_{R F}=20$ $M H z$. The amplitude of the buncher voltage is $\mathrm{V}=370 \mathrm{~V}$ b) Bunching of a beam with a momentum deviation of $\Delta \mathrm{p} / \mathrm{p}=2 \%$. The Pulse width increases to $1.7 \mathrm{~ns}$

the beam captured in the buckets and that ion losses after the phase compressor are reasonably small. The code allows also addressing e.g. the gap crossing effect, influences from space charge, etc. The $2 \pi$ phase band is divided into a number of intervals corresponding to a specific RF phase $\varphi_{i}$ with an RF voltage amplitude proportional to $\sin \left(\varphi_{i}\right)$. The beam density distribution is then calculated as the relative number of particles in each interval after crossing the drift distance between the buncher and its time focus.

At $1 \mathrm{MHz}$ RF voltage the minimum available pulse width at the position of the reaction microscope will be at least $15 \mathrm{~ns}$ and the required amplitude of the buncher voltage exceeds $5-6 \mathrm{kV}$. The resulting energy spread caused by a $1 \mathrm{MHz}$ phase compressor would thus lead to immediate loss of the beam. Increasing the RF frequency to $10 \mathrm{MHz}$ leads to bunches with a pulse width of $2 \mathrm{~ns}$ in the time focus. In this case, a voltage of $715 \mathrm{~V}$ needs to be applied to the buncher. Finally, pulses of $\tau \approx 1$ ns will become available at an operation frequency of $20 \mathrm{MHz}$, see fig. 1a. It should be pointed out that the use of the here-proposed phase gymnastics would simply be impossible at a beam energy of $E=300 \mathrm{keV}$ where the amplitude of the buncher voltage would be $21.5 \mathrm{kV}$.

Because of the low beam energy and high RF frequency, time-of-flight effects during the passage of the electric field region between the buncher electrodes are of great importance. The minimum drift tube aperture is determined by the beam size before the electron cooling and cannot be reduced to less than $20 \mathrm{~mm}$. At larger apertures there is an increased pulse width or even no bunching effect at all. Using the buncher at an RF frequency of more than $20 \mathrm{MHz}$ is not possible due to fringe field effects.

In a next step, we investigated the phase compression as a function of the beam energy spread. Simulations were performed with a well-defined deviation from the design beam energy of $20 \mathrm{keV}$, while keeping the same amplitude of the buncher voltage as for the design energy. Particles with a large momentum spread of up to $\Delta \mathrm{p} / \mathrm{p}=$ $\pm 2 \%$ were tracked through the buncher, tuned for nominal 
beam energy. In this case the pulse width increased to 1.7 ns, see fig. $1 b$.

\section{ENERGY COMPRESSOR AND HIGH HARMONIC RF CAVITY}

The evolution of a beam bunch is shown in the following fig. 2. The longitudinal emittance of the coasting beam at $\mathrm{E}=20 \mathrm{keV}$, the total bucket area and the required amplitude of the RF voltage depend on the initial energy spread of the beam and are summarized in table 2 .

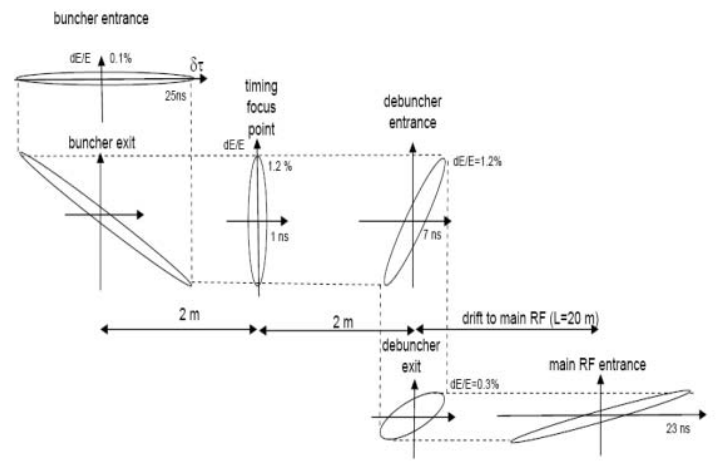

Figure 2: Sketch of the evolution of the phase space ellipse in the short pulse operation mode

The RF voltage which has to be applied to fully capture the un-cooled coasting beam is $\sim 80 \mathrm{~V}$, while it is only $\sim 1 \mathrm{~V}$ for a cooled beam.

Table 2: Coasting beam emittance and total bucket area

\begin{tabular}{|l|c|c|c|}
\hline Energy spread [dE/E] & $5 \cdot 10^{-4}$ & $5 \cdot 10^{-3}$ & $2 \cdot 10^{-2}$ \\
\hline $\begin{array}{l}\text { Longitudinal emittance of } \\
\text { coasting beam [keV·ns] }\end{array}$ & 220 & 2200 & 8800 \\
\hline RF harmonic & 440 & 440 & 440 \\
\hline Voltage to capture beam [V] & 0.8 & 82 & 1370 \\
\hline
\end{tabular}

The energy spread during the passage of the buncher/de-buncher combination is expected to be reduced from $\Delta E / E=1.2 \%$ in the time focus to $\Delta E / E=$ $3 \cdot 10^{-3}$ after the energy compressor. At a revolution period of $\sim 20 \mu$ s the bunch with a $2 \mathrm{~ns}$ pulse width will expand to 23 ns. The beam is then captured by the RF cavity for the next cycle. The bucket area must follow the growing beam emittance. For that purpose one has to provide a dynamic increase of the RF voltage amplitude in phase with increasing energy spread. The dynamic range of the RF cavity thus needs to cover variations of the voltage

Table 3. Full bucket area for an $\mathrm{E}=20 \mathrm{keV}$ beam

\begin{tabular}{|c|c|c|c|}
\hline $\begin{array}{c}\mathbf{V}_{\mathrm{RF}} \\
{[\mathrm{V}]}\end{array}$ & $\Delta \mathbf{P} / \mathbf{P}$ & $\begin{array}{c}\mathbf{2} \Delta \mathbf{E} \\
\mathrm{eV}\end{array}$ & $2 \Delta E / E_{k}$ \\
\hline 2 & $5.6 \cdot 10^{-4}$ & 24 & $10^{-3}$ \\
\hline 40 & $2.5 \cdot 10^{-3}$ & 108 & $5.3 \cdot 10^{-3}$ \\
\hline 200 & $5.6 \cdot 10^{-3}$ & 240 & $1.2 \cdot 10^{-2}$ \\
\hline 500 & $9 \cdot 10^{-3}$ & 380 & $1.9 \cdot 10^{-2}$ \\
\hline 1000 & $1.3 \cdot 10^{-2}$ & 540 & $2.7 \cdot 10^{-2}$ \\
\hline
\end{tabular}

amplitude from a few Volts to roughly $2 \mathrm{kV}$, see table 3 .

Computer simulations to build up a complete model of the USR ring short pulse operation mode are underway.

\section{SUMMARY}

The future ultra-low energy storage ring at the facility for low-energy antiproton and ion research will provide antiprotons and possibly highly charged ions with unprecedented beam quality and luminosities in the energy range of 300 down to $20 \mathrm{keV} / \mathrm{q}$.

For these experiments, shortest beam pulses in the range of 1-2 nanoseconds at lowest beam energies are necessary. Given the natural dispersion of the beam and the coupling between transverse and longitudinal motion in an electrostatic cylinder deflector, such pulses are very difficult to realize. In this paper, we present the results of a preliminary study describing a possible beam bunching scenario in the USR. The simulations indicate that even shortest pulses might be realizable if an adequate beam forming scheme is applied.

The next step will now be to extend these simulations and to build up a full model, where the beam dynamics in longitudinal and transverse phase space will be investigated in detail [10].

The generous support of the Helmholtz Association of National Research Centers (HGF) under contract number VH-NG-328 and of the Gesellschaft für Schwerionenforschung (GSI) Darmstadt is acknowledged. The authors greatly appreciate the help and useful advice of Pavel Belochitskii (CERN).

\section{REFERENCES}

[1] C.P.Welsch, M.Grieser, A.Wolf, J.Ullrich, "Layout of the USR at FLAIR", Proc. Europ. Part. Acc. Conf. Edinburgh, Scotland. (2006)

[2] C.P.Welsch, M.Grieser, J.Ullrich, A.Wolf, "An ultralow-energy storage ring at FLAIR", Nucl. Instr. and Meth. A 546 (2005) 405-417.

[3] www.oeaw.ac.at/smi/flair/TechnicalProposal/TP.htm

[4] C.P. Welsch, J. Ullrich, "FLAIR - A Facilty for LowEnergy Antiproton and Ion Research at GSI", Hyperfine Interactions 172 (1-3) (2007) 71-80.

[5] D.Fischer et al., GSI Sci. Rep. (2006).

[6] P.Schmid et al., "Preliminary Design of a Highlyflexible Extraction Scheme for the USR", this conference.

[7] C.P. Welsch, A.V. Smirnov, "Cooling rates at UltraLow Energy Storage Rings", AIP Conf. Proc. 821 (2006), 397-401.

[8] C.P. Welsch et al., "Ultra-low energy antiprotons at FLAIR", Proc. Europ. Part. Acc. Conf., Lucerne, Switzerland (2004)

[9] I.Kalagin. "Computer Code: Buncher U-400". JINR Techn.Report. 2004. 21 p.

[10]http://www.quasar.uni-hd.de quasar@kip.uni-heidelberg.de 